\title{
Committee for Establishing a Lifestyle Questionnaire for Use in Health Screenings "Lifestyle Questionnaire for Use in Health Screenings"
}

\author{
Committee Chair: Toshiyuki SHIBOSAWA \\ Members: Saburo HoRI; Takashi WADA; and Hidetoshi KASHIHARA
}

In October 2001, under the leadership of Masaki Tamura, President of the Japan Society of Health Evaluation and Promotion, the "Committee for Establishing a Lifestyle Questionnaire for Use in Health Screenings" was formed within the Japan Society of Health Evaluation and Promotion to develop a standard lifestyle questionnaire for use in multiphasic screenings.

The history of the present lifestyle questionnaire is described on pages 770-771 of Health Evaluation and Promotion, Volume 31 , Number 6 . At the $33^{\text {rd }}$ meeting of the Japan Society of Health Evaluation and Promotion held on January 28, 2005, the lifestyle questionnaire was presented in the form of a committee report. The present article concerns the details of the questionnaire.

The questionnaire consists of 38 questions in 8 categories. Points are assigned to certain questions to allow for the quantitative assessment of lifestyle and the preparation of radar charts. Questions to which points are assigned are indicated with brackets "[ ]", and point allocation is described at the end of each question.

1. Body weight: 5 questions for a total of 10 points

2. Smoking: 2 questions for a total of 20 points

3. Drinking: 3 questions for a total of 10 points

4. Dietary habits and nutrition: 14 questions for a total of 20 points

5. Physical activity and exercise: 5 questions for a total of 10 points

6. Rest: 2 questions for a total of 10 points

7. Stress: 4 questions for a total of 10 points

8. Oral hygiene: 3 questions for a total of 10 points

Total $=100$ points

1. Body weight (total: 10 points)

1.1 What is the difference in your body weight between now and six months ago?
(1) An increase of ( ) kg
(2) No change
(3) A decrease of ( ) kg
(4) Unknown

[1.2] What is the difference in your body weight between now and when you were about $18-20$ years old?
(1) An increase of ( ) kg
(2) No change
(3) A decrease of ( ) $\mathrm{kg}$
(4) Unknown

Point conversion:

0 points: a change of $\geq 20 \mathrm{~kg}$ or unknown

3 points: a change between 10 and $19 \mathrm{~kg}$

7 points: a change of $<10 \mathrm{~kg}$

1.3 At what age did you reach your maximum body weight and how heavy were at what time?

(1) At the age of ( ) years or unknown

(2) Body weight of ( ) kg or unknown

1.4.1 How do you view your figure?

(1) Skinny, (2) Thin, (3) Average, (4) Slightly overweight,

(5) Overweight

1.4.2 Do you want to change your figure?

(1) Want to lose weight, (2) Satisfied as is, (3) Want to gain weight

[1.5] How often do you weigh yourself?

(1) Less than once a month ( 0 points)

(2) 1-4 times a month (1 point)

(3) 1-6 times a week (2 points)

(4) Everyday (3 points)

2. Smoking (total: 20 points)

[2.1] Choose a statement from 1), 2) or 3) below that best describes your smoking habits, and answer the corresponding questions.

1) Never smoked

2) Have smoked in the past, but do not smoke today

Age when last smoked: ( ) years

Have not smoked for about ( ) days/months/years

Total years of smoking: about ( ) years

Number of cigarettes smoked per day: about ( ) cigarettes

Point conversion:

20 points: Never smoked and have not smoked for more than 3 years

15 points: Have not smoked for $1-3$ years

10 points: Have not smoked for less than 1 year

3) Currently smoke

Age when started to smoke regularly: About ( ) years of age

Have been smoking for: about ( ) years

Number of cigarettes smoked per day: about ( ) cigarettes

Trying to reduce smoking: yes/no

Trying to smoke lighter cigarettes: yes/no

What is your opinion on smoking?

(1) Thinking about quitting in the near future 
(2) Thinking about quitting sometime in the future, but not now

(3) Not thinking about quitting

2.2 This question is for people who do not currently smoke: How often are you exposed to second-hand smoke (passive smoking)?

(1) Hardly ever, (2) Rarely, (3) Sometimes, (4) Often

3. Drinking (total: 10 points)

[3.1] How often do you consume alcohol?

(1) Do not drink or drink less than once a week

(2) Drink about ( ) days a week

Point conversion:

10 points: Do not drink or less than once a week

[3.2] How much alcohol do you consume in a week? Indicate type and quantity of alcohol that you consume in a typical week.

Beer: Large: ( ) bottles

Medium $(500 \mathrm{ml})$ : ( ) cans/bottles

Small $(350 \mathrm{ml})$ : ( ) cans/bottles

Sake: ( ) cups

Distilled spirit: ( ) glasses

Wine: ( ) glasses

Whisky or brandy: ( ) shots

Others: ( ) shots

Point conversion:

Weekly alcohol consumption is calculated based on the type and amount of alcohol and the number of days.

10 points: $0-154 \mathrm{~g}$

7 points: $155-462 \mathrm{~g}$

2 points: $463-846 \mathrm{~g}$

0 points: $\geq 847 \mathrm{~g}$

[3.3] This question is for people who drink alcohol. Choose one of the following answers that best describes you.

(1) Currently reducing my alcohol consumption

(2) Planning to reduce my alcohol consumption in the near future

(3) Thinking about reducing my alcohol consumption, but can not

(4) Not thinking about reducing my alcohol consumption Point conversion:

3 points: The level of weekly alcohol consumption is $\geq 463 \mathrm{~g}$, and is currently reducing the amount of alcohol consumption.

4. Dietary habits and nutrition (total: 20 points)

[4.1] How fast do you eat your meals?
(1) Slow (2 points)
(2) Average (2 points)
(3) Fairly fast (1 point)
(4) Very fast ( 0 points)

[4.2] How often do you eat until you feel full?
(1) Hardly ever (3 points)
(2) Occasionally ( 2 points)
(3) Frequently (1 point)
(4) Every meal (0 points)

[4.3] How often do you eat at regular times?
(1) Always (1 point)
(2) Mostly (0 points)
(3) Rarely (0 points)
(4) Never (0 points)

[4.4] How many days a week do you eat breakfast?
(1) 7 days (1 point)
(2) 4-6 days ( 0 points)
(3) 1-3 days ( 0 points)
(4) 0 days ( 0 points)

[4.5] How many nights a week do you eat out?
(1) 0 days ( 1 point)
(2) 1-3 days ( 1 point)
(3) 4-6 days ( 0 points)
(4) 7 days (0 points)

[4.6] How many hours after begging dinner do you normally go to bed?
(1) $\geq 3$ hours ( 1 point)
(2) 2-3 hours (1 point)
(3) 1-2 hours (0 points)
(4) $\leq 1$ hour ( 0 points)

[4.7] How many nights a week do you eat something after dinner?
(1) 0 days ( 1 point)
(2) 1-3 days (0 point)
(3) 4-6 days ( 0 points)
(4) 7 days ( 0 points)

[4.8] Do you eat balanced meals?
(1) Always (2 points)
(2) Sometimes (1 point)
(3) Rarely (0 points)
(4) Never (0 points)

[4.9] What amount of snacks or drinks that contain sugar do you have?

(1) None or a small amount (2 points)

(2) Average amount (1 point)

(3) Slightly more than average (0 points)

(4) Plenty (0 points)

[4.10] What amount of fatty food (fried food and stir-fry) do you have?

(1) None or a small amount (2 points)

(2) Average amount (1 point)

(3) Slightly more than average (0 points)

(4) Plenty (0 points)

[4.11] How salty do you like to have your food taste?
(1) Not salty (1 point)
(2) Average (1 point)
(3) Slightly salty (0 points)
(4) Very salty ( 0 points)

[4.12] What amount of vegetables do you eat?

(1) Plenty (1 point)

(2) Average amount (1 point)

(3) Slightly less than average (0 points)

(4) Almost none (0 points)

[4.13] Do you look at nutritional information when eating out or buying food? 

(1) Always (1 point)
(2) Frequently (1 point)
(3) Rarely (0 points)
(4) Hardly ever (0 points)

[4.14] Do you eat calcium-rich food (dairy products, soybean, seaweed, small fish with bones, etc.)?

(1) Every meal (1 point)

(2) Frequently (1 point)

(3) Rarely (0 points)

(4) Hardly ever (0 points)

5. Physical activity and exercise (total: 10 points)

[5.1] Do you feel like you lack exercise?

(1) No, I don't lack exercise (1 point)

(2) No, I probably don't lack exercise (1 point)

(3) Yes, I may lack exercise (0 points)

(4) Yes, I strongly feel lack exercise (0 points)

[5.2.1] How many minutes a day do you walk (including work and commuting)? ( ) minutes

Point conversion:

2 points: $\geq 60$ minutes

1 point: $20-59$ minutes

0 points: $<20$ minutes

[5.2.2] During the above, how many minutes a day do you consciously walk fast? ( ) minutes

Point allocation:

2 points: $\geq 10$ minutes

1 point: $1-9$ minutes

0 points: 0 minutes

[5.3] Besides work, do you do exercise that makes you sweat?

(1) ( ) times a week

( ) minutes per exercise ( 2 points)

(2) Less than once a week (1 point)

(3) Seasonal (1 point)

(4) None (0 points)

[5.4] What is your level of daily physical activity (activities of daily living, including work and exercise)?

(1) Very active (3 points)

(2) Fairly active (2 points)

(3) Fairly inactive (0 points)

(4) Inactive ( 0 points)

If you answered either (1) or (2), please move to Question 6.

5.5 This question is for people who answered either "Fairly inactive" or "Inactive" to the question above: Choose one of the following answers that best describes your physical activity.

(1) Currently trying to increase my level of physical activity

(2) Thinking about increasing my level of physical activity in the near future

(3) Thinking about increasing my level of physical activity, but can not

(4) Not thinking about increasing my level of physical activity
6. Rest (total: 10 points)

[6.1] Do you think that you get a sufficient amount of rest?

(1) Sufficient (5 points)

(2) Mostly sufficient (4 points)

(3) Often insufficient (1 point)

(4) Insufficient (0 points)

[6.2.1] Do you think that you sleep a sufficient number of hours?

(1) Sufficient (5 points)

(2) Almost sufficient (4 points)

(3) Rather insufficient (1 point)

(4) Insufficient (0 points)

6.2.2 How many hours do you sleep and what time do you go to bed?

Sleep about ( ) hours a night

Go to bed about ( ) each night (use the 24-hour clock ("military time"): for example, 11:00 pm at night is 23:00).

7. Stress (total: 10 points)

[7.1] Do you wake up refreshed in the morning?
(1) Always (4 points)
(2) Often (2 points)
(3) Sometimes (2 points)
(4) Never (0 points)

[7.2] How often do you feel that you have accumulated stress?
(1) Never (6 points)
(2) Sometimes (4 points)
(3) Frequently (2 points)
(4) Always (0 points)

7.3 Have you recently experienced a change in the level of stress at work or in your personal life?
(1) Decreased
(2) Unchanged
(3) Increased

7.4 Have you experienced a high level of stress over the last 12 months? (e.g., death of a family member, divorce, job transfer, job change, etc.)
(1) Yes
(2) No

8. Oral hygiene (total: 10 points)

[8.1] How many times a day do you brush your teeth?
(1) $\geq 3$ times ( 6 points)
(2) 2 times (4 points)
(3) 1 time (3 points)
(4) Not even once on some days (0 points)

[8.2] Do you floss or use an interdental brush?
(1) Everyday (1 point)
(2) Occasionally (1 point)
(3) Never (0 points)
(4) Wear dentures (1 point)

8.3 Do you see a dentist regularly?
(1) Yes (3 points)
(2) No (0 points) 